\title{
Influence of the robotic exoskeleton Lokomat on the control of human gait: an electromyographic and kinematic analysis
}

\author{
Student: Filipe Barroso* \\ Advisor: C. Santos \\ University of Minho, Azurém \\ Guimarães \\ Portugal \\ *email: fbarroso@dei.uminho.pt
}

\author{
Co-Advisor: J. C. Moreno \\ Grupo de Bioingeniería, Consejo Superior de \\ Investigaciones Científicas \\ Crta Campo Real Km 0,200. Arganda del Rey, Madrid \\ Spain
}

\begin{abstract}
Nowadays there is an increasing percentage of elderly people and it is expected that this percentage will continue increasing, carrying huge organizational costs in rehabilitation services. Recent robotic devices for gait training are more and more regarded as alternatives to solve cost-efficiency issues and provide novel approaches for training. Nevertheless, there is a need to address how to target muscular activation and kinematic patterns for optimal recovery after a neurological damage. The main objective of this work was to understand the underlying principles that the human nervous system employs to synchronize muscular activity during walking assisted by Lokomat. A basic low-dimensional locomotor program can explain the synergistic activation of muscles during assisted gait. As a main contribution, we generated a detailed description of the electromyographic and biomechanical response to variations in robotic assistance in intact humans, which can be used for future control strategies to be implemented in motor rehabilitation.
\end{abstract}

Index Terms- Human gait, Motor control, Neurorehabilitation, Electromyography, Exoskeleton

\section{INTRODUCTION}

Nowadays, there is still a lack in robotic therapy, because it is not designed and adapted according to the muscular coordination of the patient. As a main contribution, this Master Thesis tests a hypothesis about the underlying principles that the human nervous system may employ to synchronize muscular activity during walking assisted by a rehabilitation exoskeleton. Theoretical formulations and experimental evidences in this regard are presented in the last part of this section. To introduce the reader to the key points, this section also refers the procedures and the employed equipment to record electric activity from the muscles, as well as the kinematics and kinetics during the human walking.

\section{A. Electromyography}

All the movements people perform during their daily life are a result of different mechanisms in the Nervous System. The Nervous System is divided into the Central Nervous System (CNS), constituted by the brain and the spinal cord, and the Peripheral Nervous System (PNS), constituted by the nerves. Motor commands generated in the CNS are sequences of electric signals, called action potentials, which travel through the nerves in direction to the effectors (muscles or glands). Muscle tissue conducts actions potentials in a similar way nerves do, which finally results in the muscular contractions. These potentials propagated along the muscular tissues are signals that can be recorded by a method called electromyography (EMG). Surface electromyography (sEMG) is electromyography using surface electrodes, a method worldwide used nowadays [1]. Electromyography has many possible clinical and biomedical applications [1], including the exploration of the physical integrity of the motor system and the study of muscle activation during walking.

\section{B. Kinematics and Kinetics}

Kinematics is the branch of Classical Mechanics that describes the movement of objects or groups of objects. Understanding the kinematics of human movement is of great value to evaluate functional performance of limbs under normal and abnormal conditions.

Kinetics is the study of the forces acting on a system, as the human body for example. In this work, it was analyzed the interaction forces between an external device and each subject during robotic-aided walking.

\section{Rehabilitation and Assistive Devices}

Strokes and spinal cord injuries constitute alterations of the normal function of the nervous system and lead to abnormal generation of motor commands responsible for movement control. Even though this phenomenon destroys nervous cells and connections, the nervous system can be trained (rewired) to recruit new circuits, a phenomenon also known as neural plasticity. Motor rehabilitation after an event like a stroke, for example, can spark plasticity, modulating cortical organization, and in the most successful cases leading to a recovery of the damaged/lost functions.

The number of people with disabilities in the lower limbs is growing. This demographic change will impose a higher overload in health care to deal with the risks associated with the aging [2]. Robotic devices are a candidate solution to solve the majority of those issues and allow the older people to maintain their independence and their quality of life. Therefore, there is a huge potential on using robotic devices for motor rehabilitation purposes. Individuals who received body-weight supported treadmill training after a stroke or spinal cord injury got better electromyographic activity during 
locomotion and also obtained better recovery results than the others who received conventional gait training [3] [4]. Robotic devices can automate and repeat the trainings, and with unlimited duration of time, representing a more effective and a cheaper form of rehabilitation. Robotic devices for rehabilitation purposes can be divided in static devices and portable exoskeletons or orthoses. Only static devices will be considered in this work.

Static robotic devices for rehabilitation purposes (usually found in motor rehabilitation clinics), guarantee safety, repeatability, unlimited duration of training and adapt the gait to the type of patient and pretended training [5]. Hocoma AG created the Lokomat, a robotic exoskeleton to automate the motor training of lower limbs, which is commercialized all over the world. Lokomat is composed by a treadmill and a body-weight support system. It has four degrees of freedom, allowing the movement control of hip (one degree of freedom in the left hip and other in the right hip) and knees (one degree of freedom in the left knee and other in the right knee) in the sagittal plane.

One important feature of Lokomat is the applied guidance force (GF - the amount of aid the patient receives during the walking). A value of $100 \%$ of guidance force corresponds to a strict guiding (position control with stiff Lokomat joints) of the exoskeleton. A value of $0 \%$ corresponds to free run mode (easily moveable Lokomat joints). Reducing the guidance force allows the user to move more freely and actively, i.e., the user can move away from the defined gait pattern. Providing too much assistance (or guidance force) can have negative consequences for motor learning [6]. What may be important for rehabilitation purposes is to provide assistance as needed or in other words, to assist the patent only as much as is needed to accomplish the tasks.

\section{Modular organization of the Nervous System}

Actual studies have been trying to understand how the central nervous system produce the neuronal responses corresponding to the planned movements, coordinating a large number of degrees of freedom of the musculoskeletal system [7] [8] [9] [10]. Actual evidences suggest that the nervous system controls motor tasks by using a low-dimensional modular organization of muscle activation constituted by motor modules and activation signals [11] [12]. Recent computer simulations [8] showed that some motor tasks (including walking) can be produced through the coordinated activation of few synergies, each one associated with specific biomechanical subtasks. This modular control is represented in figure 1 .

This modular organization can be thought as a neuronal network in which the activation signals are generated in some brain structures according to the sensory information received about the different tasks to be performed. Then, the activation signals are directed to the motorneurons via a premotor network (it can be located in the brainstem or in the spinal cord) that specifies the relative weight of each activation signal in each muscle. The relative weight of each activation signal is given by the respective motor module (that specifies the weight of the respective activation signal in the muscles).
The set of an activation signal and the respective motor modules is called synergy. In summary, each muscle synergy receives as input a modulation signal from higher neural centers, and gives as output a weighted activation signal to a set of muscles. The activation of each muscle results in a weighted sum of all the synergies connected with that muscle, multiplied by the neural commands. This mechanism permits to control the high dimensional space of muscular activations by means of a lower dimensional set of neural commands.

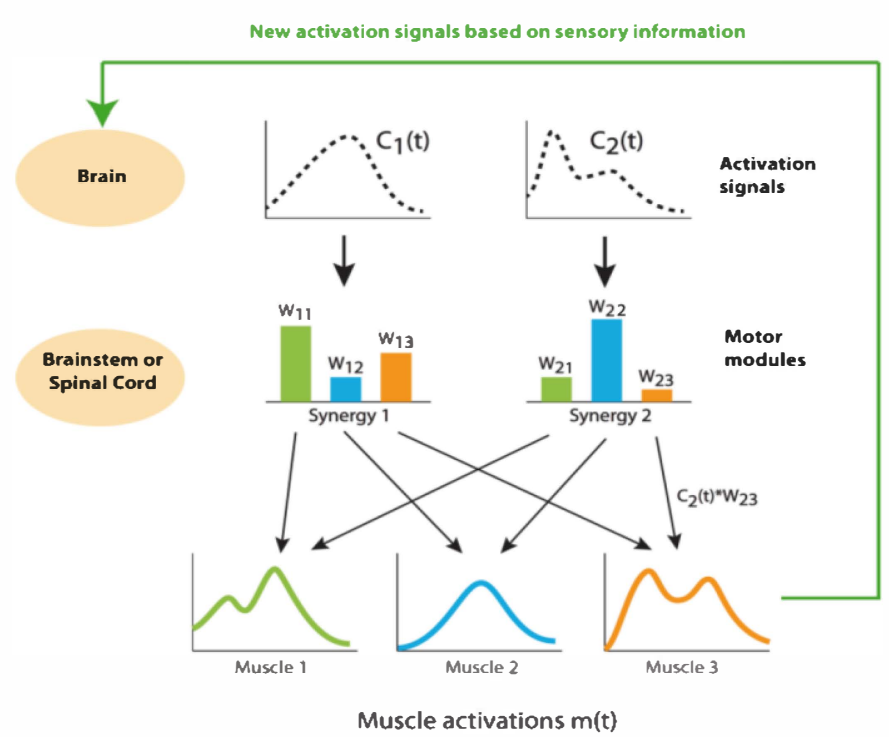

Figure 1. Theory of the modular control presented by nervous system to control movements. Each activation signal activates a specified synergy with a multiply factor $\mathrm{Ci}$, that can be a function of time or type of movement. Each synergy is composed of weighted activations (motor modules) for each muscle. Therefore, each muscle's tuning curve is a weighted average of the activations of each synergy.

If researchers can identify all the synergies, how they change according to the different motor tasks performed and according to the type of impairment of each person, it can be possible to develop focused therapy to train the damaged modules. The comprehension of this modular organization will be useful for movement restoration by using, for example, FES if few modules can describe several functional tasks.

\section{MOTIVATION}

This Master Thesis had three main objectives: 1) to study the muscular electric activity during walking in Lokomat, by varying the total assistance provided by the device, as well as the walking speed; 2) to analyze kinematic changes obtained during Lokomat-assisted walking, as well as the interaction forces between each user and the robotic device; 3) to understand how this modular organization of the nervous system involved in the synchronization of the muscular activity works during walking assisted by Lokomat. Only healthy subjects participated in the study.

\section{METHODS}

\section{A. Participants}

Eight healthy participants ( 6 males and 2 females; age $=$ $25,75 \pm 4,37$ years; body weight $=69,5 \pm 9,84 \mathrm{Kg}$; height $=$ 
$1,76 \pm 0,08)$ with no neurological injuries or gait disorders participated in the study. The participants had no previous experience with robotic-assisted walking. A local committee provided ethic approval for this study.

\section{B. Procedures}

The participants were asked to walk on the Lokomat robotic orthosis, after being fitted to the robotic orthosis and secured by a safety harness. The participants were asked to walk at speeds of $1.5,2.0$ and $2.5 \mathrm{Km} / \mathrm{h}$ speed and robotic GF was set at $100 \%, 70 \%, 40 \%$ and $20 \%$ with a fixed body weight unloading level of $30 \%$. This value of BWS was regarded as a reasonable value to enable comfortable walking with the robotic orthosis at higher speeds that might represent more challenges to the volunteers. Each walking trial lasted 60 seconds. The participants were instructed to follow the robotic guidance aided by the Lokomat's visual representation of biofeedback values. The visual biofeedback values, designed to motivate the patient to improve the walking performance, were displayed step-by-step in line graphs representing the walking performance over the last steps. In particular, the participants were instructed to follow the robotic movements in order to maintain a constant biofeedback value during each trial. All the combinations of speed and guidance forces were recorded after a familiarization interval of 60 seconds for each combination. In addition, treadmill walking at speeds of 1.5 , 2.0 and $2.5 \mathrm{Km} / \mathrm{h}$ speed was measured with every participant. The ten central gait cycles in each condition were selected for analysis.

Bipolar electrodes (Ag- $\mathrm{AgCl}$, Fiab S.p.A.) were fastened to specific locations to record EMG from the rectus femoris $(\mathrm{RF})$, vastus lateralis (VL), semitendinosus (ST), biceps femoris (BF), gastrocnemius medialis (GM), gastrocnemius lateralis (GL) and tibialis anterior (TA) of the dominant leg of each participant, using a wireless EMG acquisition system (BTS Pocket EMG, Myolab). Skin sites were determined following the SENIAM recommendations and shaved and cleaned with alcohol. Data were wirelessly streamed during the treadmill and robotic-guided walking conditions and analyzed using Matlab 7.0 (The Mathworks, Natick, MA) and SPSS statistical software (v. 18.0 IBM).

In the robotic-guided walking condition, the knee and hip angles and the forces at the knee and hip joints were recorded from the analog output of the Lokomat. In the treadmill walking condition an electrogoniometer was fixed to measure the knee angle in the sagittal plane. In the robotic-guided and treadmill walking conditions a footswitch was placed beneath the heel of the dominant leg and the status of the contact of the heel with the ground was extracted applying a threshold to its analog signal. The resulting binary signal was used for stride identification and segmentation in gait cycles.

\section{EMG signal analysis}

Raw EMG data was band-passed filtered (1st order zerolag Butterworth digital, pass-band 20-400 Hz) to attenuate DC offset, motion artifacts and high frequency noise. EMG signals were smoothed using a 50-point root mean squared (RMS) algorithm. The smoothed EMG signals were then averaged per each stride in order to obtain an average cycle with 1000 points. Signals were time-interpolated to 101 samples and normalized by their maximal value per each stride. The normalized EMG signals were computed to obtain an average of the group for further analysis, for each muscle and condition. For each subject and for the average of the group, the EMG signals of each condition were combined into an $\mathrm{m} \mathrm{x}$ $\mathrm{t}$ matrix $\left(\mathrm{EMG}_{0}\right)$, where $\mathrm{m}$ indicates the number of muscles (seven muscles in this case) and $t$ is the time base (101 values that represents the gait cycle from $0 \%$ until $100 \%$ ) [7].

An NNMF algorithm [7] was applied to the $\mathrm{m} x \mathrm{t}$ matrix for extraction of motor modules from each subject for each condition. A priori, the number of modules and activation signals, n, was specified (dimensionality two, three and four), and the NNMF algorithm found the properties of the modules by populating two matrices: an $\mathrm{m} \times \mathrm{n}$ matrix, which specifies the relative weighting (motor modules) of a muscle in each activation signals, and an $\mathrm{n} \times \mathrm{t}$ matrix, which specifies the activation timing of each activation signal. These two matrices were multiplied to produce an $\mathrm{m} \times \mathrm{t}$ matrix $\left(\mathrm{EMG}_{\mathrm{r}}\right)$ in an attempt to reconstruct the EMG signals. $\mathrm{EMG}_{\mathrm{r}}$ was compared to $E M G_{0}$ by calculating the sum of the squared errors $\left(\mathrm{EMG}_{0}-\right.$ $\left.\mathrm{EMG}_{\mathrm{r}}\right)^{2}$ and the result was used for iterative optimization until it converged on the motor modules and the activation timings of the activation signals that minimized the error. The variability accounted for (VAF) was calculated to determine the minimum number of activation signals needed to adequately reconstruct $\mathrm{EMG}_{0}$ of each subject and of the average of the group. The VAF was calculated as the ratio of the sum of the squared error values to the sum of the squared $\mathrm{EMG}_{0}$ values $\left[\mathrm{VAF}=1-\left(\mathrm{EMG}_{0}-\mathrm{EMG}_{\mathrm{r}}\right)^{2} / \mathrm{EMG}_{0}\right]$. VAF was calculated for each muscle and for each condition within the gait cycle. In order to ensure the quality of reconstructed signals within each region of the gait cycle, VAF was also calculated within seven phases of the gait cycle: 1) initial double support, 2) mid stance, 3) terminal stance, 4) pre swing, 5) initial swing, 6) mid swing and 7) terminal swing. We analyzed the VAF results from the computed activation signals from the average EMG of the group. A minimal VAF value of $80 \%$ in each gait cycle portion was required to consider the reconstruction quality satisfactory. Preliminary testing led to exclude dimensionality five since inclusion of a 5th module did not improve the reconstruction significantly for the analysis.

\section{Kinematics and force analysis}

Kinematic and force data was averaged per each stride in order to obtain data time normalized, expressed as a percentage of the total gait cycle, i.e., 0 to $100 \%$.

The angular range of motion (ROM) in the sagittal plane for hip and knee was computed by subtracting the minimum joint angle from the maximum joint angle for Lokomat trials for each condition of GF and speed. The ROM in the sagittal plane for knee during the treadmill walking was also calculated, for each condition of speed. The time (\% of gait cycle) at which the minimum and maximum angles were obtained, for all conditions, were also determined. 
The kinetic range of forces (ROF) in the hip and knee joints of the Lokomat was found by subtracting the minimum joint force from the maximum joint force for robotic-guided walking trials for each condition of GF and speed and also for each gait phase.

\section{E. Statistical analysis}

The differences in motor modules and activation signals across subjects for treadmill and robotic-guided walking, and for each subject in robotic-assisted walking were tested using a three -factor ANOVA and Tukey's post hoc analysis.

The differences inter-subject and intra-subject variability of activation signals and differences in average motor modules between treadmill and robotic-guided walking were tested using a Spearman's correlation.

\section{RESULTS AND DISCUSSION}

\section{A. Modular organization comparing Treadmill with Lokomat walking}

Four modules were required to reconstruct the EMG envelops with VAF superior than $80 \%$ for all muscles and gait phases. This result supports previous studies reporting the same number of modules [7] [8] [11] [12].

As an example, the computed motor modules, activation signals and EMG envelopes for all the conditions of Treadmill and Lokomat walking at $1.5 \mathrm{Km} / \mathrm{h}$ are represented on figure 2 . The extracted motor modules and activation signals revealed that the activity of each muscle consisted in contributions from each module, but it is usually dominated by a single module (except Rectus femoris and Vastus lateralis).

For all the conditions of speed and guidance force, the modular control presented the following characteristics:

- Synergy 1 consisted mainly of flexor activity from the Rectus femoris (hip flexor) and activity of the Vastus lateralis (knee extensor). This synergy was mainly active during the early stance phase.

- Synergy 2 mostly consisted of activity of the Semitendinosus (knee flexor) and Biceps femoris (hip extensor) muscles at late swing and early stance.

- Synergy 3 consisted mainly of activity of the Gastrocnemius medialis and Gastrocnemius lateralis (ankle plantarflexors) and this synergy was primarily active during late stance.

- Synergy 4 consisted mainly of activity of the Tibialis anterior (ankle dorsiflexor). This synergy was mainly active during early stance and early swing.

Motor modules values were significantly similar both on treadmill and Lokomat, whereas the activation signals varied much more.

From all conditions analyzed in this study, the activation signals and the correspondent motor modules in Lokomat walking at $1.5 \mathrm{Km} / \mathrm{h}$ and with $20 \%$ GF presented lower correlation values in relation to the other conditions. This result was expected, because it was a very 'robotized gait' (see

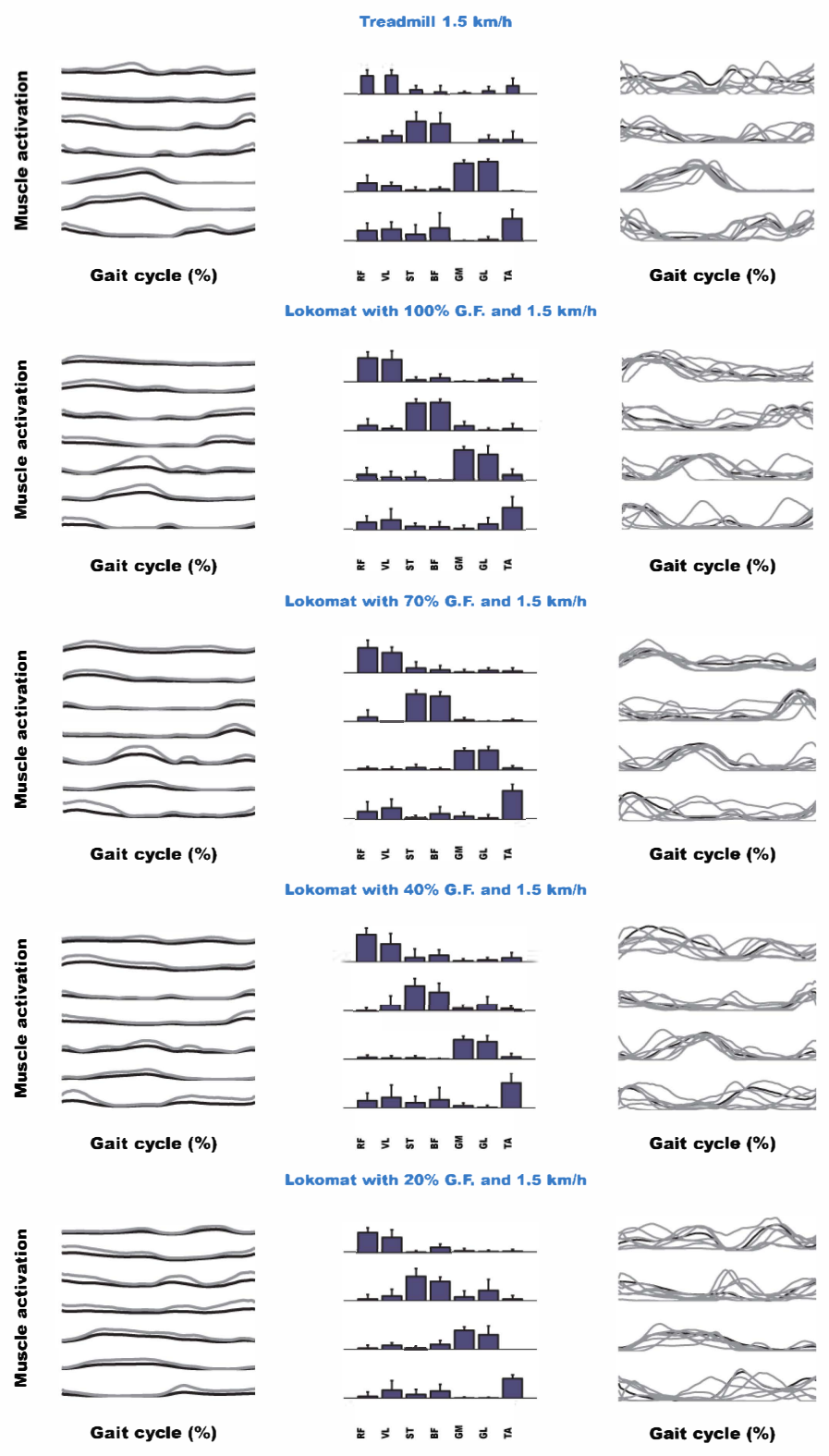

Figure 2. Synergies obtained for all the conditions of Treadmill and Lokomat walking using $1.5 \mathrm{Km} / \mathrm{h}$. (A) Average (black lines) and standard deviation (gray lines) of the EMG envelopes of the seven muscles for all the conditions using $1.5 \mathrm{Km} / \mathrm{k}$. (B) Average motor modules and (C) the correspondent activation signals. Thin gray lines represent the results of each individual of the study, whereas the thick black lines represent the group average.

kinematic pattern in figure 4) and also because individuals mentioned discomfort while walking with this combination of force and speed. In relation to the other conditions, it was possible to observer that the computed motor modules and activation signals of the trials using $40 \%$ and $20 \%$ of GF presented higher correlation values with the results from treadmill, more than the results from $100 \%$ and $70 \%$ of GF compared with treadmill. This fact supports the idea that walking with less GF would conduct to similar activation signals and motor modules to the obtained in treadmill, for healthy subjects. 


\section{B. Muscular activation}

Average EMG envelopes recorded from the seven muscles, for both types of walking and for all conditions of guidance force and speed, are illustrated in Figure 3. Different muscular activation patterns were obtained according to the demand. (a)

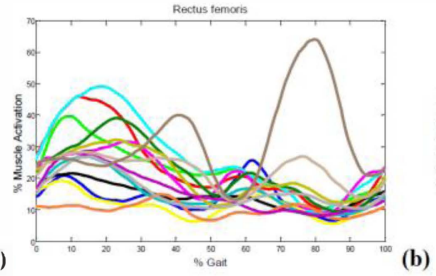

(c)
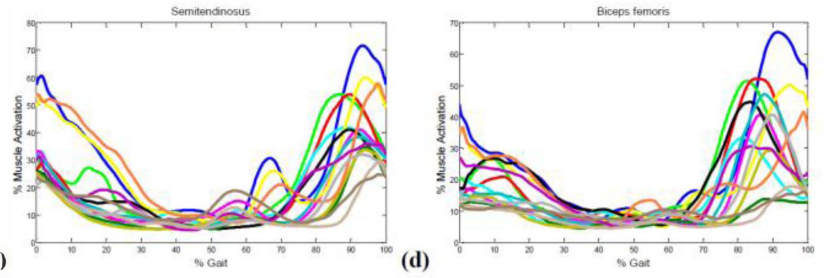

(e)
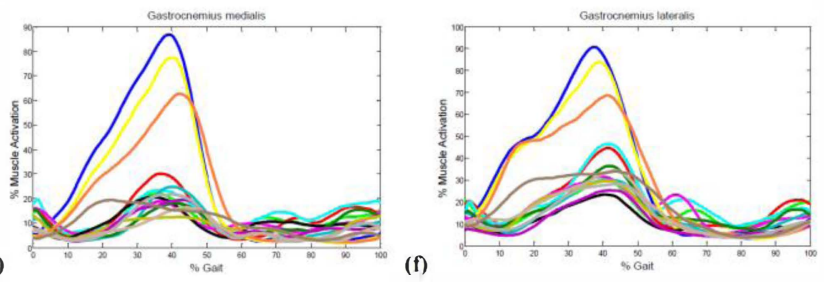
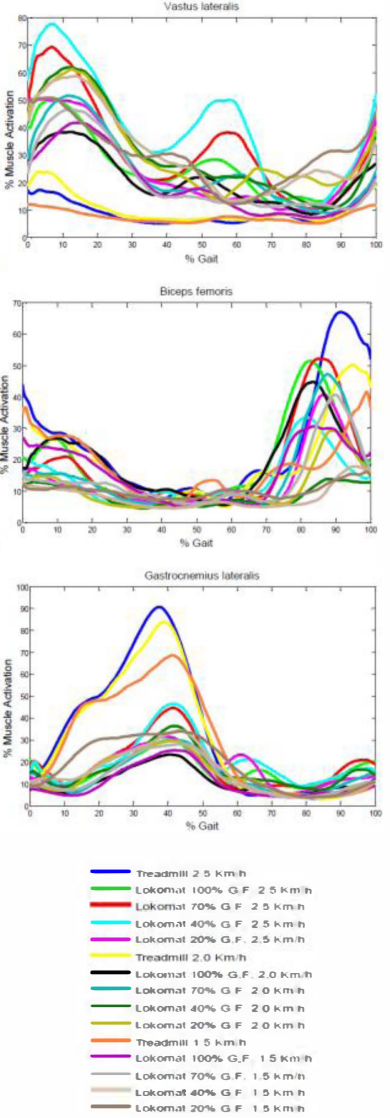

(g)

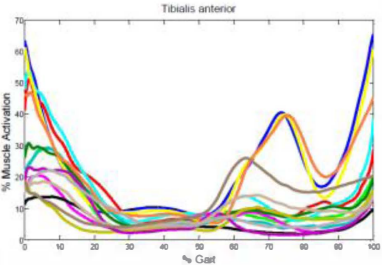

Figure 3. Average envelope signals of Rectus femoris (a), Vastus lateralis (b), Semitendinosus (c), Biceps femoris (d), Gastrocnemius medialis (e), Gastrocnemius lateralis (f) and Tibialis anterior, for all the conditions of guidance force and speed (all subjects).

In general, muscular activation increased with the increase of speed, for all conditions of GF used in Lokomat and for treadmill walking. Generally, there was also higher muscular activation for $20 \%$ and $40 \%$ GF in respect to the other guidance forces, for all conditions with the same speed.

Activation patterns of Rectus femoris and Vastus lateralis presented higher activity during robotic-guided walking than treadmill walking, for all the variations of guidance force and speed. There was significantly less contribution of Gastrocnemius medialis, Gastrocnemius lateralis and Tibialis anterior to the mechanical demand imposed during roboticguided walking than treadmill walking. In general, the hamstrings (Semitendinosus and Biceps femoris) presented similar activation patterns for both types of walking.

In relation to gastrocnemius medialis, gastrocnemius lateralis and tibialis anterior activity during Lokomat walking, Hidler [5] reported that the drop in muscle activity (he only used $100 \%$ of guidance force) could be related with the use of footlifters, which assist ankle dorsiflexion for toe clearance during swing phase. Interestingly, we obtained less muscular activity not only using $100 \%$ of guidance force, but also for the other guidance forces. In relation to the quadriceps (Rectus femoris and Vastus lateralis), Hidler explained that people usually rotate their hips and also abduct their legs to allow the toe to clear the floor, which are movements quite limited to perform in Lokomat. Therefore, participants of his study (just using $100 \%$ of guidance force) exerted higher muscle activity in the quadriceps to help in the elevation of the feet and preventing toe from getting caught in the treadmill. Interestingly, we obtained higher activity for all the guidance forces.

\section{Kinematics}

In order to analyze if the participants would change joint trajectories as a response to the altered mechanical demand, we examined the average knee and hip joints trajectories and the corresponding ROM (range of motion) in the sagittal plane. Figure 4 represents the average knee and hip angular trajectories, for all the conditions of robotic-aided walking.
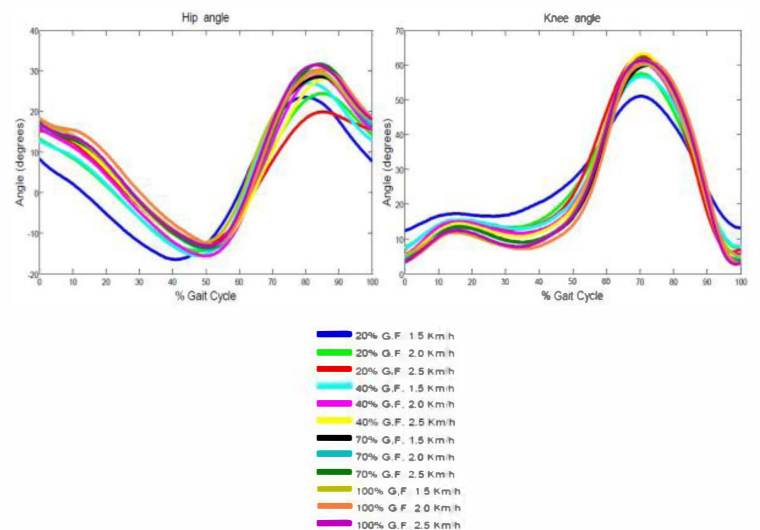

Figure 4. Mean kinematic trajectories of the hip and knee joints (sagittal plane) during the gait cycle in the robotic-aided walking condition.

Angular patterns and the correspondent ROM were very similar for all conditions, except for the condition of $20 \% \mathrm{GF}$. Nevertheless, despite being firmly strapped into the Lokomat, a small amount of variance was found. This happened, because each participant can produce relative movement in relation to the Lokomat, although Lokomat guides the limbs of all subjects through pre-programmed trajectories. In general, the ROM decreased with the decreasing amount of GF.

The ROM of the hip joint, using $20 \%$ GF, was smaller when compared with the other conditions of guidance force, for the same walking speed. The ROM of the knee joint using $20 \% \mathrm{GF}$ and $1.5 \mathrm{Km} / \mathrm{h}$ was smaller when compared with the other conditions. In general, it was found that the ROM of both knee and hip joints increased with the speed and the \% GF, except for the condition of $20 \% \mathrm{GF}$, where the hip ROM decreased with the increase of speed.

\section{Force (Kinetics)}

In general, subjects were able to walk with a similar kinematic pattern imposed by the robot. But changes in the 
mechanical pattern, related with the changes in modular control and induced by altered demand, were observed (Figure 5). In general, the ROF (Range Of Forces) decreased with the decrease of GF and the increase of speed.
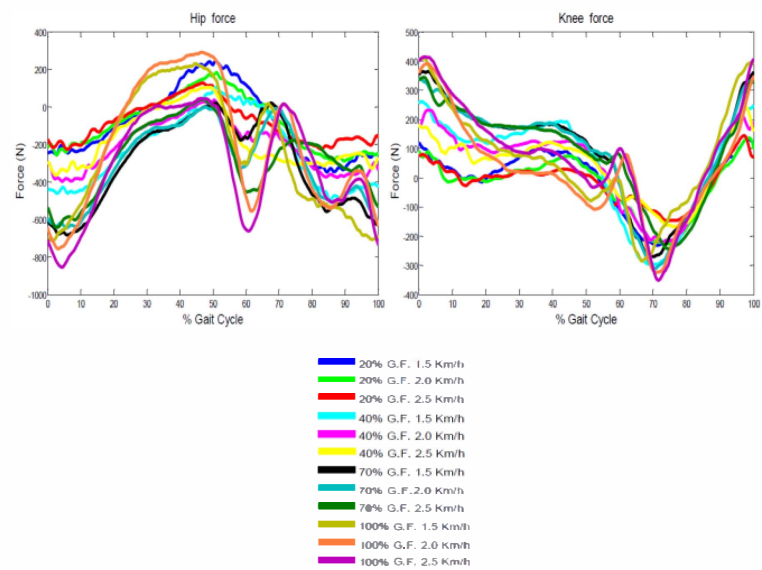

Figure 5. Mean interaction joint forces between the participants and Lokomat during the gait cycle.

Main deviations across combinations in the interactions forces were found in the transition from stance to swing.

For the hip joint, we observed that with $20 \%$ and $40 \%$ GF, as the leg moved to prepare the swing motion and initiate it, relative hip extension and flexion torques were small. Nevertheless, for higher GF ( $70 \%$ and $100 \%)$, the hip torque patterns required a more complex strategy as subjects exerted significantly higher hip flexion torques at mid-swing. This reveals a strategy that is adopted to pull the leg towards swing that is accentuated with augmented mechanical demand. This behavior correlates with the increased RF (hip flexor) activity and decreased activity of the hamstrings (hip extensor).

For the knee joint, the ROF decreased with the decrease of GF and the increase of speed. The ROF using $20 \%$ and $40 \%$ GF is reduced when compared to higher levels of GF. The main differences in forces across combinations for this joint were observed in the transition from stance to swing. For $20 \%$ and $40 \% \mathrm{GF}$, the limb produced reduced extension torques during pre-swing, followed by reduced flexion torques at initial swing. In turn, using $70 \%$ and $100 \%$ GF resulted in increased knee extension torques at pre-swing followed by increased knee flexion torques at initial swing. This behavior correlates with the increased RF (knee extensor) and VL (knee extensor) activity during the stance phase.

\section{CONCLUSIONS}

This study evaluated the effects of robotic-aided walking in healthy participants. It was developed a protocol to analyze the differences in the modular organization of the nervous system, in the muscular activation, as well as the kinematic and kinetic differences between normal walking and walking assisted by an exoskeleton, changing the guidance force and the speed. The results of our study are very important, because they provide a baseline for comparison with future studies about motor rehabilitation in post-stroke patients during robotic therapy, as well as to develop new rehabilitation methods.

Future design control strategies to be implemented in robotic gait trainers might be directed to promote similar modular control to the obtained in this study. Robotic devices to retrain human gait after brain damage should be adapted to train the nervous system to induce the required timing of activity generated by central pattern generator neurons that is directed to the motorneurons.

\section{ACKNOWLEDGMENTS}

This study has been founded by the European Commission, project BETTER contract number FP7-ICT-2009-247935 and also by the Spanish Ministry for Science and Innovation, in the framework of the project HYPER 'Hybrid Neuroprosthetic and Neurorobotic Devices for Functional Compensation and Rehabilitation of Motor Disorders' (Ref. CSD2009-00067).

This work was also funded by FEDER Funds through the Operational Programme Competitiveness Factors - COMPETE and National Funds through FCT - Foundation for Science and Technology under the Project: FCOMP-01-FEDER-0124022674.

\section{REFERENCES}

[1] M. B. Raez, M. S. Hussain, and Mohd F. Yasin. Techniques of EMG signal analysis: detection, processing, classification and applications. Biological procedures online, 8:11-35, 2006.

[2] F. Barroso, A. Frizera, C. Santos, and R. Ceres. Revisão crítica das ortóteses activas para membros inferiores. VI Congresso Iberoamericano de Tecnologías de Apoyo a la Discapacidad, 2:369-377, 2011.

[3] S. Hesse and D. Uhlenbrock. A mechanized gait trainer for restoration of gait. Journal of rehabilitation research and development, 37(6):701-708, 2000.

[4] A. Wernig, A. Nanassy, and S. Müller. Laufband (treadmill) therapy in incomplete paraplegia and tetraplegia. J Neurotrauma, 16(8):719-26, 1999.

[5] J. M. Hidler and A. E. Wall. Alterations in muscle activation patterns during robotic assisted walking. Clin Biomech (Bristol, Avon), 20(2):184-193, February 2005.

[6] Laura Marchal-Crespo and David J. Reinkensmeyer. Review of control strategies for robotic movement training after neurologic injury. Journal of NeuroEngineering and Rehabilitation, 6(1):6-20, June 2009.

[7] David J. Clark, Lena H. Ting, Felix E. Zajac, Richard R. Neptune, and Steven A. Kautz. Merging of healthy motor modules predicts reduced locomotor performance and muscle coordination complexity post-stroke. J Neurophysiol, 103(2):844-857, February 2010.

[8] Richard R. Neptune, David J Clark, and Steven A Kautz. Modular control of human walking: a simulation study. Journal of Biomechanics, 42(9):1282-1287, 2009.

[9] Andrea D'Avella, Philippe Saltiel, and Emilio Bizzi. Combinations of muscle synergies in the construction of a natural motor behavior. Nature Neuroscience, 6(3):300-8, 2003.

[10] G. Cappellini, Y. P. Ivanenko, R. E. Poppele, and F. Lacquaniti. Motor patterns in human walking and running. J Neurophysiol, 95(6):34263437, June 2006.

[11] R. R. Neptune and C. P. McGowan. Muscle contributions to whole-body sagittal plane angular momentum during walking. J Biomech, 44(1):612, January 2011

[12] L. Gizzi, J.F. Nielsen, F. Felici, Y.P. Ivanenko, and D. Farina. Impulses of activation but not motor modules are preserved in the locomotion of subacute stroke patients. J Neurophysiol, 106(1):202-210, 2011. 